\title{
The quest for self-consistency in hydrogen bond definitions
}

\author{
Diego Prada-Gracia*, Roman Shevchuk*, and Francesco Rao ${ }^{\dagger}$ \\ Freiburg Institute for Advanced Studies, School of Soft Matter Research, \\ Albertstrasse 19, 79104 Freiburg im Breisgau, Germany
}

(Dated: October 29, 2018)

\begin{abstract}
In the last decades several hydrogen-bond definitions were proposed by classical computer simulations. Aiming at validating their self-consistency on a wide range of conditions, here we present a comparative study of six among the most common hydrogen-bond definitions for temperatures ranging from $220 \mathrm{~K}$ to $400 \mathrm{~K}$ and six classical water models. Our results show that, in the interval of temperatures investigated, a generally weak agreement among definitions is present. Moreover, cutoff choice for geometrically based definitions depends on both temperature and water model. As such, analysis of the same water model at different temperatures as well as different water models at the same temperature would require the development of specific cutoff values. Interestingly, large discrepancies were found between two hydrogen-bond definitions which were recently introduced to improve on more conventional methods. Our results reinforce the idea that a more universal way to characterize hydrogen-bonds in classical molecular systems is needed.
\end{abstract}

\section{INTRODUCTION}

Hydrogen bonding represents a fundamental interaction in molecular systems [1. Its peculiarity resides in the common aspects it has with both covalent bonds and van der Waals interactions. The strong directionality together with the ease of being formed and broken at ambient conditions makes it an important ingredient in water structure and dynamics 2, protein stability 3 and ligand binding 4. Notwithstanding, a universal definition of this interaction is still missing $[5$.

Hydrogen-bonds are formed between two polar atoms via a hydrogen which is covalently bound to one of the two. This interaction is highly directional. For example, in bulk water at $300 \mathrm{~K}$ the angle $\mathrm{OH}-\mathrm{O}$ is mostly below 30 degrees [6], while the donor-acceptor distance is of around $3.5 \AA$ [7. Despite the apparent simplicity, the presence of thermal fluctuations as well as the non-trivial effects of the environment made the development of an operative definition of this bond difficult.

In the last decades, several definitions were proposed based on computer simulations 8]. The most popular ones look at bond formation by using a mixture of distances and angles between the two partners [9 11]. Others tried to avoid altogether cutoffs by proposing topology-based definitions 12 14. Given the many degrees of freedom involved in molecular association, it is now clear that all definitions retain some degree of arbitrariness [15].

In most cases, hydrogen-bond definitions were developed at specific thermodynamic conditions. However, not much is known on the behavior of those definitions as a function of temperature and water model. In this

\footnotetext{
*these authors contributed equally (in alphabetical order) ${ }^{\dagger}$ Corresponding author; E-mail: francesco.rao@frias.unifreiburg.de; Phone: +49 (0)761 203 97336, Fax: +49 (0)761-203 97451
}

research paper, we present a critical assessment of six classical hydrogen-bond definitions based on the analysis of molecular dynamics simulations of water in a temperature range spanning from $220 \mathrm{~K}$ to $400 \mathrm{~K}$. Six among the most widespread classical water models were used in the analysis, including SPC 16, SPC/E 17, TIP3P 18, TIP4P [19], TIP4P-Ew [20] and TIP4P/2005 21]. Our investigation elucidates, in a statistical way, common features and limitations of current classical hydrogen-bond definitions.

This work is an effort to present a transparent comparison between hydrogen-bond definitions in several different conditions, including temperature, water model and cutoff dependence. Scientist willing to know the consequences of choosing one method or the other will find our results a good starting point for further development.

\section{METHODS}

\section{A. Hydrogen-bond definitions}

Six hydrogen-bond definitions were considered. They can be classified into two broad classes: geometrical and topological (Fig. 1). An important difference between the two is that geometrical definitions make use of cutoffs on inter-atomic distances and angles while the latter mostly avoid this problem. A brief description of the definitions follows.

\section{Geometrical definitions}

1. $r_{O H}$. In this definition the oxygen-hydrogen distance $\left(r_{\mathrm{OH}}\right)$ is used as criterion (Fig. 1A) 10. In the original work, a cutoff of $2.3 \AA$ was proposed by simulating amorphous ice at $\mathrm{T}=10 \mathrm{~K}$ with the TIPS2 potential [? ]. The distance cutoff value is related with the position of the first minimum in the oxygen-hydrogen radial distribution function. 
A

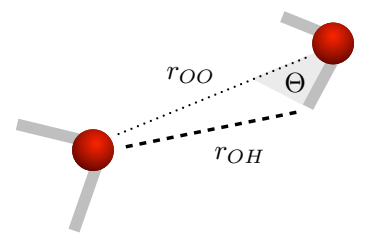

geometrical

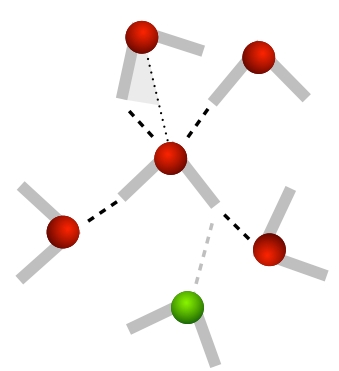

topological
B

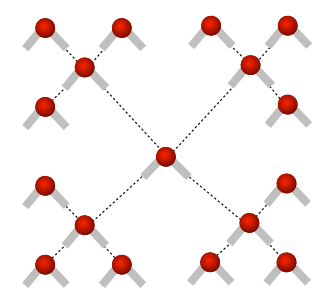

$P_{4}$ configuration

FIG. 1: (A) Hydrogen-bond definitions can be roughly partitioned into two classes: geometrical and topological. (B) A water molecule with a fully-coordinated first and second solvation shells is shown as $P_{4}$ configuration, see Methods for details.

2. $r_{O O} \Theta$. This definition makes use of both the oxygen-oxygen distance $\left(r_{O O}\right)$ and the $\angle O O H$ angle $(\Theta)$ between two water molecules. In the original work, a bond was considered formed when $r_{O O}$ and $\Theta$ were smaller than $3.5 \AA$ and 30 degrees, respectively [11]. The distance cutoff was taken from the position of the first minimum in the oxygenoxygen radial distribution function. Missing a clear signature of the bond state in the distribution of the angle $\Theta$, the cutoff value was taken from experimental data [6, 7].

3. Sk. The hydrogen-bond definition of Skinner and collaborators is based on an empirical correlation between the occupancy $N$ of the $O \cdots H \sigma^{*}$ orbital and the geometries observed in molecular dynamics simulations [9]. Two water molecules were considered bonded if the value of $N$ is higher than a certain cutoff which is taken in correspondence to the position of the first minimum in the distribution of $N$. In the original paper a cutoff equal to 0.0085 was chosen by analyzing MD simulations of the SPC/E model at ambient conditions.

\section{Topological definitions}

4. $D \Theta$. A hydrogen-bond is formed between a hydrogen atom and its nearest oxygen not covalently bound. An additional restriction was imposed: the angle $\Theta$ had to be lower than $\pi / 3$. In the original work [13, this definition was applied to the study of the $\mathrm{SPC} / \mathrm{E}$ water model for temperatures ranging from 273 to $373 \mathrm{~K}$.

5. DA. Two criteria for the hydrogen-bond were used: (i) the acceptor is defined as the closest oxygen to a donating hydrogen and (ii) this hydrogen is the first or second nearest neighbor of the oxygen. As a consequence, the total number of hydrogen-bonds per water is limited to four. This definition was proposed with simulations of the EMP water model at $292 \mathrm{~K}[12$.
6. TP. A hydrogen-bond is formed between a hydrogen and its closest oxygen. When more than one hydrogen-bond between the two water molecules is found, the one with the shortest oxygen-hydrogen distance is considered to be formed [14. This definition was mainly evaluated at ambient conditions using the TIP4P/2005 water model.

The six hydrogen-bond definitions above as well as all the analysis tools applied in this work were implemented in the software AQUA-LAB freely distributed at raolab.com.

\section{B. Water structural propensities including the second shell}

Hydrogen-bond propensities up to the second solvation shell were obtained by calculating the following parameters: the probability $P_{4}$ to have a water molecule with a fully-coordinated first and second solvation shells, for a total of 16 bonds (right panel of Fig. 11; and the probability to have four $\left(P_{4}^{*}\right)$, three $\left(P_{3}\right)$ and two or less $\left(P_{210}\right)$ bonds with a generic first solvation shell. In the calculation of $P_{4}^{*}$ the propensity of $P_{4}$ was subtracted. A more comprehensive study of these four propensities, including temperature and water model dependence, was already presented elsewhere [23].

\section{Hydrogen-bond kinetics}

Kinetics was analyzed in terms of hydrogen-bond lifetime distributions. The lifetime was calculated as follows. For each definition, pairwise hydrogen bonds among all water molecules were calculated for every frame. For each of the water pairs that formed a bond, the time span for how long that particular bond lasted is called lifetime. The distribution was then calculated by building an histogram of all the lifetimes collected in the molecular trajectory. The average lifetime was denoted with the symbol $\tau$. 


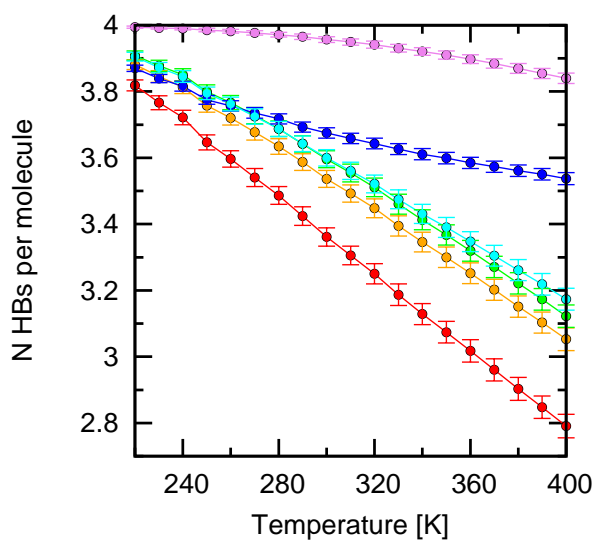

FIG. 2: Average number of hydrogen bonds per water molecule for the six hydrogen-bond definitions: $r_{O H}$ (orange), $r_{O O} \Theta$ (green), $S k$ (red), $D \Theta$ (cyan), DA (blue) and TP (purple)

\section{Molecular dynamics simulations}

All molecular dynamics simulations (MD) were run with GROMACS 24. The setup included a cubic water box of 1024 water molecules. Temperature and pressure were controlled with the velocity rescale thermostat 25 and Berendsen barostat [26, respectively. In both cases a 1 ps relaxation time was used. Non-covalent interactions were treated with a $1 \mathrm{~nm}$ cutoff and Particle-Mesh-Ewald 27. Each water model was simulated for 1 ns after a 10 ns equilibration run, saving coordinates every 4 fs for a total of 250'000 snapshots per model.

For the present analysis six popular classical water models, namely SPC [16], SPC/E [17, TIP3P [18, TIP4P [19], TIP4P-Ew [20] and TIP4P/2005 [21] were simulated for temperatures ranging from $220 \mathrm{~K}$ to $400 \mathrm{~K}$ at ambient pressure.

\section{RESULTS}

\section{A. Hydrogen-bond propensities in temperature space}

MD simulations of 1024 SPC/E water molecules were performed for temperatures ranging from $220 \mathrm{~K}$ to $400 \mathrm{~K}$ at ambient pressure. For each condition the average number of hydrogen-bonds per molecule was calculated. Results for the six definitions under study (see Methods for details) are shown in Fig. 2. As expected, the general trend shows a decreasing number of hydrogen-bonds per molecule by increasing temperature. At $220 \mathrm{~K}$ this number was very similar among all approaches ranging from 3.8 to 4.0. However, at ambient temperature definitions deviated quite considerably. $S k$ provided the smallest number of bonds (3.37 per molecule), while, with an average connectivity of 3.95 , the purely topological definition
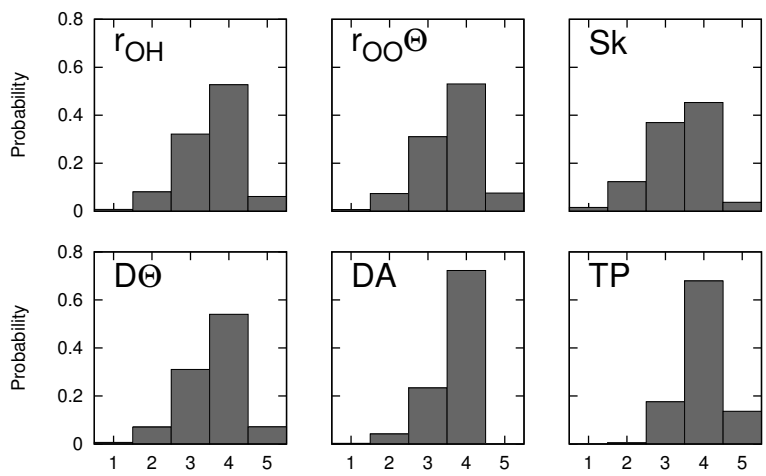

FIG. 3: Average number of bonded partners for the six hydrogen-bond definitions at $300 \mathrm{~K}$.

$(T P)$ provided the largest number. This discrepancy amplified as the temperature was further increased to $400 \mathrm{~K}$ with values approaching 2.79 and 3.84 for $S k$ and $T P$, respectively. Better agreement along the whole temperature range was found among $r_{O H}, r_{O O} \Theta$ and $D \Theta$. It is interesting to note that among these definitions, $S k$ was optimized against ab-initio calculations using the SPC/E model at 300K (same model as here). At least in principle, Skinner's data should provide the most accurate estimation at $300 \mathrm{~K}$ and ambient pressure.

Discrepancies were also found in the distribution of the number of bonded partners (Fig. 3). At 300K geometrical definitions were quite consistent among each other, with a larger fraction of three hydrogen-bonded configurations for $S k$. For the topological case, $D A$ and $T P$ agreed on the number of four coordinated molecules. However, the former detected a larger fraction of three and two bonded molecules while $T P$ presented a non-negligible fraction of cases with five partners and no evidence for two bonded molecules. This scenario changes when a topological definition is coupled with an angle cutoff $(D \Theta)$. In this case, almost identical results as the conventional $r_{O O} \Theta$ were found with an agreement that persists in the entire temperature range as shown in Fig. 2 (green and cyan data).

In Fig. 4 hydrogen-bond propensities including the second solvation shell are presented. The behavior of these propensities strongly depend on the hydrogen-bond definition taken into account. Consistency was found within two groups. The first one includes $r_{O H}, r_{O O} \Theta$ and $D \Theta$ and the second one TP and $D A$. Sk did not match very well any of them. The value of $P_{4}$, i.e., the probability to have a four-coordinated water molecule with a fully coordinated first and second shells (Methods and Fig. 1B), was equal to 0.34 and 0.58 at $220 \mathrm{~K}$ for $S k$ and $T P$, respectively (red data). As temperature was increased this difference became even more pronounced. A similar disagreement was also observed for the other three propensities.

An interesting case is given by $P_{4}^{*}$. This quantity reports on four-coordinated water molecules with an arbi- 

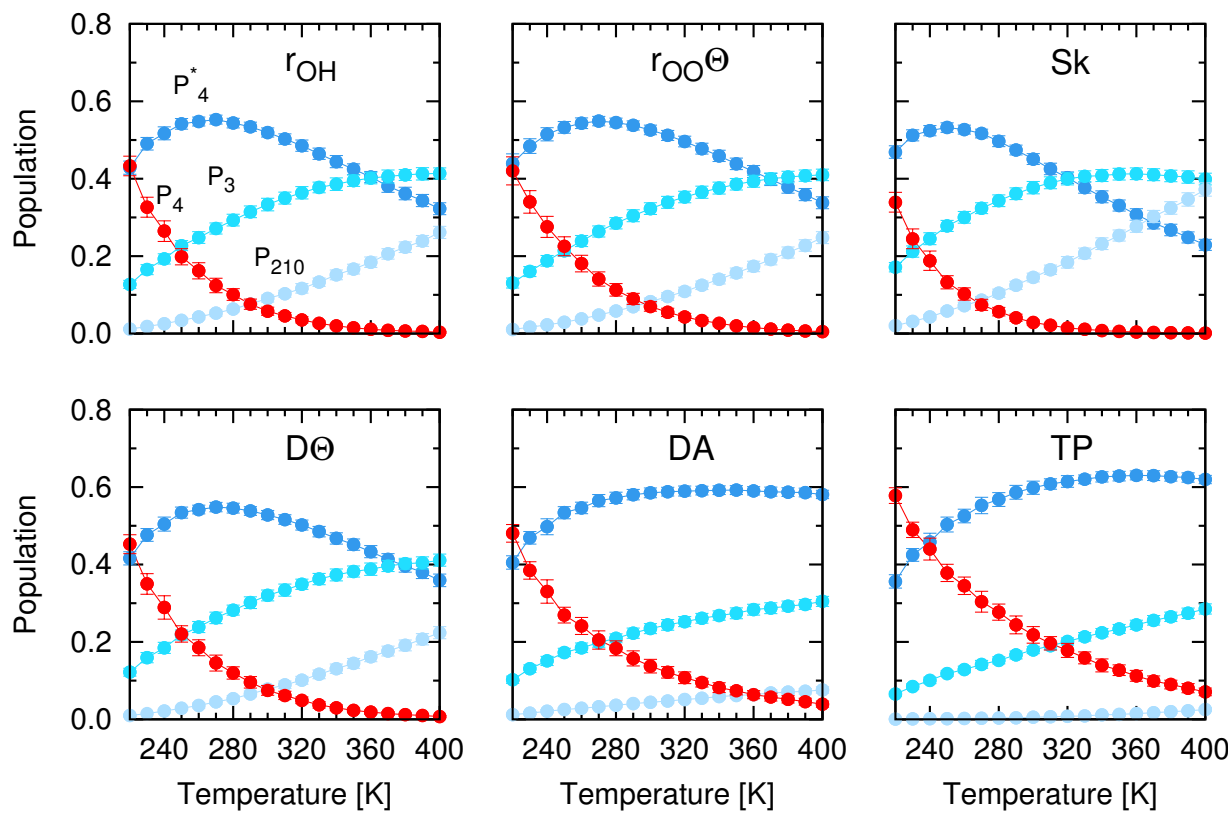

FIG. 4: Hydrogen-bond propensities including the second solvation shell for temperatures between $220 \mathrm{~K}-400 \mathrm{~K}$. $P_{4}, P_{4}^{*}, P_{3}$ and $P_{210}$ are shown in red, blue, light blue and very light blue, respectively (see Methods for details).

trarily disordered second solvation shell. For all definitions this quantity presented a peak. However, $T P$ and $D A$ made an exception being the maximum much more shallow and at a higher temperature with respect to the other approaches. This leads to an over estimation of four coordinated water molecules which are predicted to be the most abundant configuration at temperatures as high as $400 \mathrm{~K}$. This result is counter intuitive as waters with three or less hydrogen-bonds would have been expected to represent a larger fraction of the sample at a such high temperature. Substantial discrepancies among definitions were also found in the case of $P_{210}$ (water molecules with two bonds or less). For the case of $T P$ this probability was essentially zero at all temperatures while it grew with temperature in all the other cases.

\section{B. Hydrogen-bond lifetime}

Water kinetics was analyzed through hydrogen-bond lifetimes (see Methods). Distributions for the six hydrogen-bond definitions at 300K are shown in Fig. 5A. Fastest decays (i.e. shorter life times) were observed for $r_{O O} \Theta$ (green) and $D \Theta$ (cyan), strongly suggesting that fluctuations along the $\Theta$ angle represent the major responsible for the faster kinetics. On the other hand, the largest lifetimes were found with the $T P$ definition. At very short times $(<200 \mathrm{fs})$ purely topological approaches provided the best results (inset of Fig. 5A). In fact, both
$D A$ (blue) and TP (purple) showed a smooth decay, in contrast to all the other definitions which provided a debatable oscillating behavior 28. This observation strongly suggests that those fluctuations are an artifact of the use of cutoffs.

For the average lifetime $\tau$, Arrhenius behavior in the range $260 \mathrm{~K}<\mathrm{T}<400 \mathrm{~K}$ was found, breaking down for lower temperatures (Fig. $5 \mathrm{~B}) . r_{O O} \Theta, D \Theta$ and $r_{O H}, D A$, $T P$ provided fastest and slowest timescales, respectively. $S k$ shifted from one group to the other while changing temperature (red data in Fig. 5B).

\section{Self-consistency}

Hydrogen-bonds were described so far on the base of propensities and kinetics. Now, we investigate the robustness of the geometrical definitions with cutoff choice. The aim of the following analysis is to understand what is the influence of temperature and water model on the distributions relevant to cutoff choice. In fact, default cutoff values were originally proposed from experiments and calculations at specific temperatures and water models (see Methods). Although in most cases prescriptions were given to properly choose the cutoffs, default values were often applied in conditions far away from the original works.

First, temperature dependence was investigated. For $r_{O H}$, the distribution that matters is the oxygen- 

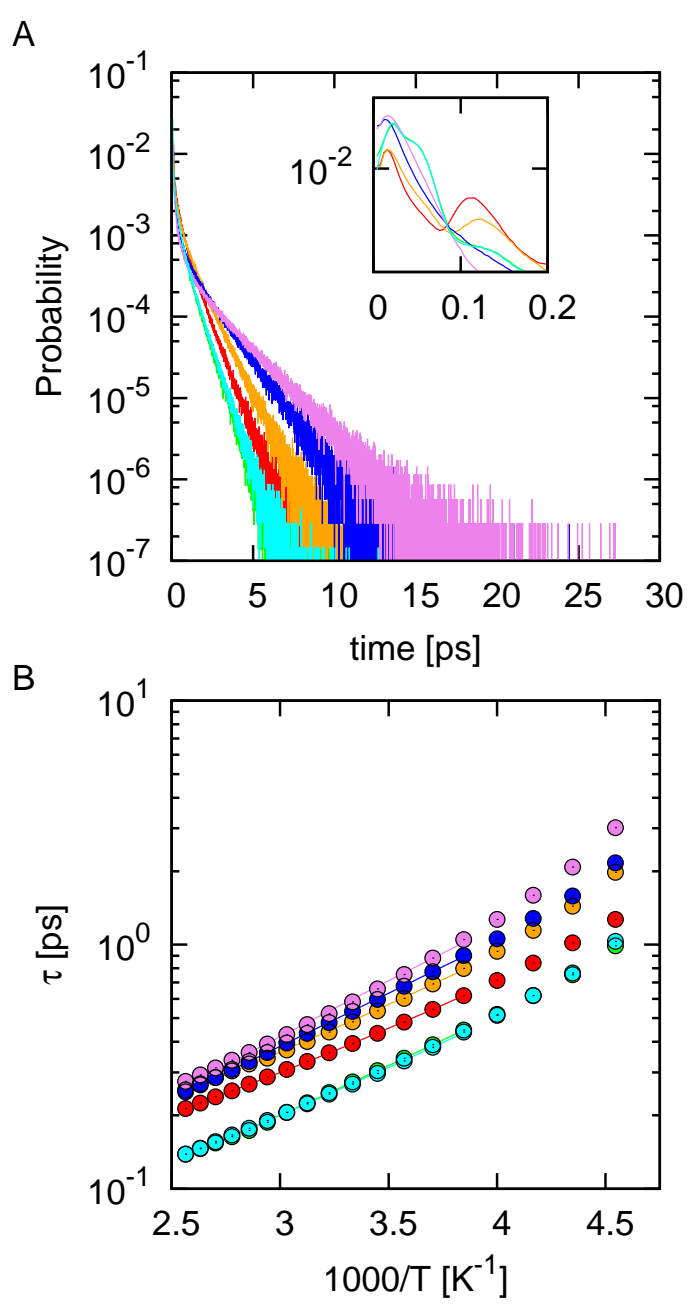

FIG. 5: Hydrogen-bond kinetics for the six different definitions: color-code is the same as in Fig. 2. (A) The lifetime distribution at $\mathrm{T}=300 \mathrm{~K}$ is shown. (B) the average hydrogenbond lifetime versus $1 / \mathrm{T}$ is plotted (error bars are smaller than the symbol size). The Arrhenius behavior is observed in the range of temperatures from 260 to $400 \mathrm{~K}$.

hydrogen radial distribution function $(g(r)$, left column in Fig. 6). The plot shows that the first minimum becomes less pronounced with temperature while its position gets closer to the origin (from 2.424 to $2.419 \AA$, left middle row of Fig. 6). Choosing the cutoff according to the position of the minimum, the average number of hydrogen-bonds per molecule was significantly affected despite the small change of the cutoff value. In the bottom left panel of Fig. 6 the difference between a "standard" cutoff approach (empty circles) and a temperature dependent cutoff (filled circles) is shown.

Similar results were obtained for the other two geometrical definitions. For these cases the value of the cutoff was chosen according to the position of the first minimum of the oxygen-oxygen radial distribution function and the distribution of the occupancy $N$ for the case of $r_{O O} \Theta$ and $S k$, respectively (second and third columns of Fig. 6). Lacking of a bimodal behavior we intentionally avoided the study of the angle $\Theta$ cutoff dependence.

Interestingly, radial distribution functions depend not only on temperature but also on the water model under study. This suggests a further dependence on cutoff choice. To verify this idea, we ran MD simulations of six of the most commonly used water models (see Methods for details). In Fig. 7 results for the $S k$ definition are presented. The data reports on the position of the first minimum in the occupancy $N$ distribution as a function of temperature for different water models. According to the original prescription 9, the hydrogen-bond cutoff should be taken as the position of this minimum. The plot shows that this value strongly depends on both water model and temperature. Similar conclusions can be drawn for the case of $r_{O H}$ and $r_{O O}$.

\section{DISCUSSION}

Despite the fundamental role of hydrogen bonding in molecular processes, a robust, self-consistent and universally accepted definition is still missing. In the context of classical molecular simulations several proposals emerged in recent years. In this work, we systematically analyzed six among the most popular of these definitions, trying to elucidate their range of validity, strengths and limitations. The definitions under study can be classified into two main groups: geometrical and topological.

Geometry-based methods make use of cutoffs. The latter identify a geometrical volume where the hydrogenbond is thought to be formed. However, the numerical value of a cutoff is usually chosen with some degree of arbitrariness. We found that the distributions behind the definitions of a cutoff are strongly dependent on temperature and water model. As a consequence, hydrogen-bond cutoffs would need careful evaluation for each temperature and water model in order to be self-consistent with the underlying distributions. Unfortunately, the use of optimal cutoffs do not necessarily lead to improved consensus among hydrogen-bond definitions. The cutoff optimization presented here is similar in spirit to an interesting prescription recently suggested by Skinner and co-workers [9]. In that case, hydrogen-bond boundaries were defined by the isoline passing through the saddle point of bond forming/breaking of the corresponding coordinates taken into account (e.g. $R_{O O}$ and $\Theta$ ). This approach is certainly optimal when the free-energy projection onto those coordinates represents an accurate description of the system kinetics. However, given the large number of degrees of freedom involved, this assumption does not hold in general 29 33. Looking at the recrossings present on top of the barrier for hydrogen-bond forming/breaking as well as the oscillations observed in the lifetime distribution at short times suggest that those maps do not describe the kinetics well. 

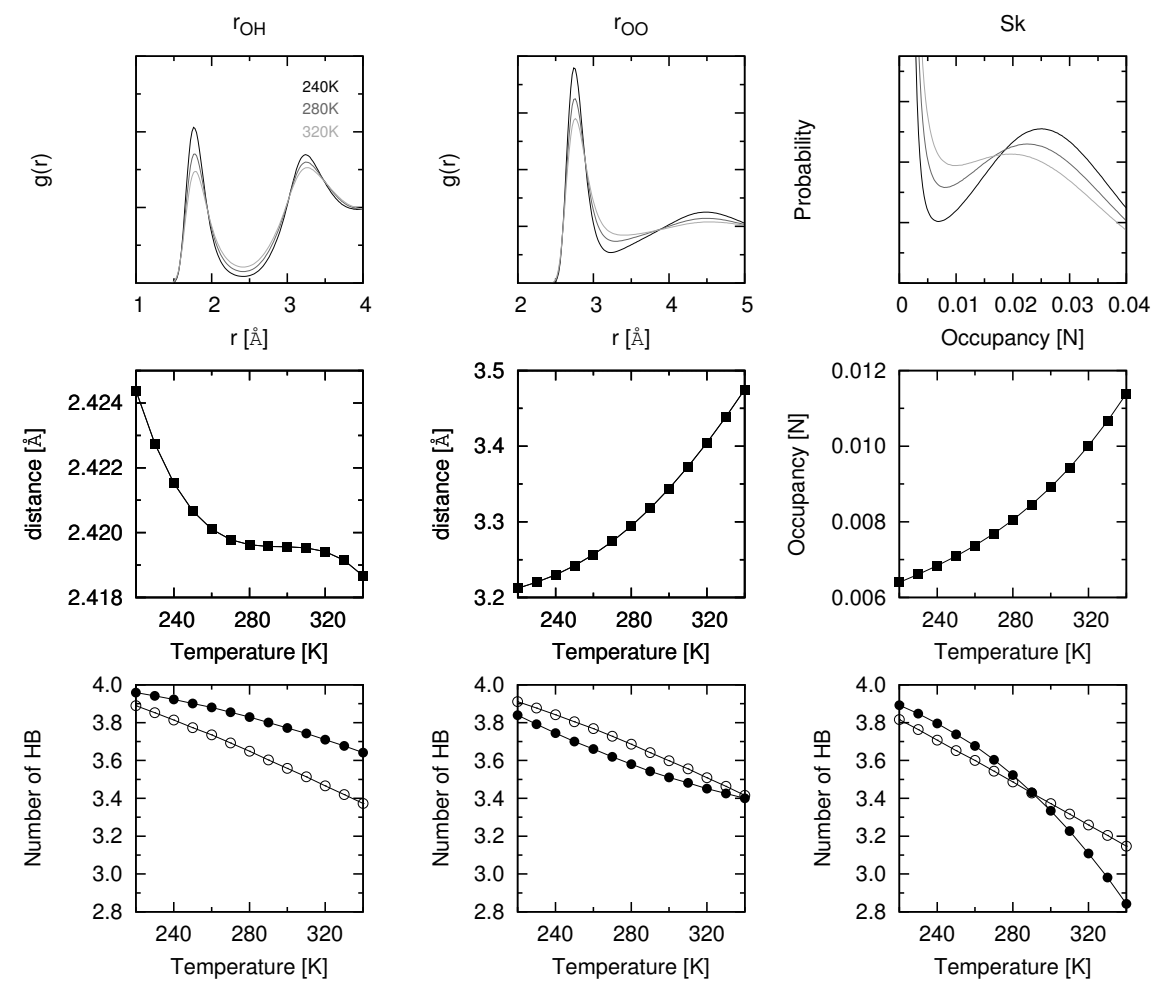

FIG. 6: Temperature dependence for cutoff choice. Data relative to the $r_{O H}, r_{O O} \Theta$ and $S k$ definitions are shown in the first, second and third column, respectively. (Top) The oxygen-hydrogen, oxygen-oxygen radial distribution functions and the occupancy distribution are displayed from left to right. (Middle) Cutoff dependence as a function of temperature. (Bottom) Average number of hydrogen bonds with fixed and variable cutoffs are shown as empty and filled circles, respectively.

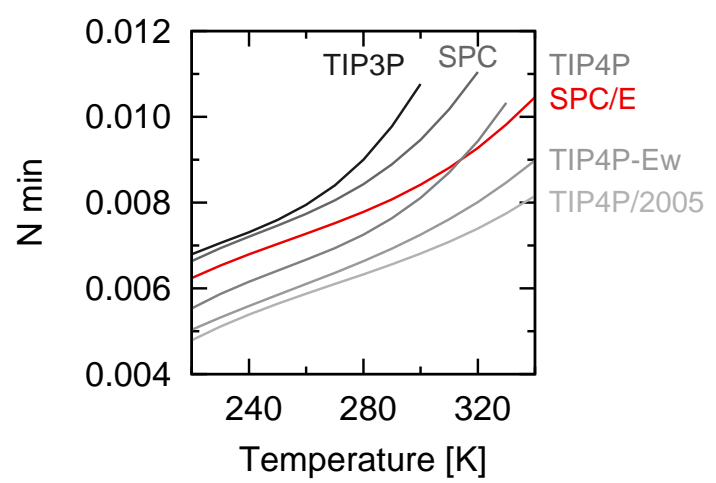

FIG. 7: The position of the first minimum of the occupancy distribution relative to the $S k$ definition for different water models. Red points refer to the SPC/E model which was used for the rest of the analysis presented in this paper.

To cure some of the issues related to a cutoff based approach, topology-based definitions were recently introduced [12, 14]. However, these approaches are characterized by a very small number of broken hydrogen bonds at high temperatures: a trend that seems to be debatable.

Overall, our analysis put in evidence a number of limitations in current approaches, highlighting a general lack of consensus among them. Somewhat surprising was to find that two of the most recent definitions, $S k$ and $T P$, were the ones to agree the least with each other. This certainly motivates the exploration of alternative routes, like the use of multi-body definitions going beyond the classical pairwise models [34, 35. However, we speculate that the most overlooked player in this game might very well be kinetics. Our suggestion is motivated by the recent approaches emerged in the study of protein conformational changes which constructively used system kinetics to characterize the underlying free-energy landscape. Based on configuration-space-networks [30, 31] and Markov-state-models [33, 36, 37] those methods provided projection-free state definitions and interconversion rates. As such, two structures are detected to belong to the same state if their dynamics is similar, shifting the problem from being geometrically similar to be kinetically similar. Applications of these ideas to the study of bulk water were recently put forward by our group [32, 38, but a straightforward extension to hydrogenbond structure and dynamics is not trivial. Given the fundamental role of hydrogen bonding in molecular systems, we believe that such type of developments will obtain increased attention in the future. 


\section{ACKNOWLEDGMENT}

This work is supported by the Excellence Initiative of the German Federal and State Governments.

[1] G. Jeffrey, An introduction to hydrogen bonding, vol. 12 (Oxford University Press New York, 1997).

[2] C. Fecko, J. Eaves, J. Loparo, A. Tokmakoff, and P. Geissler, Science 301, 1698 (2003).

[3] I. K. McDonald and J. M. Thornton, J. Mol. Biol. 238, 777 (1994).

[4] R. Wade, K. Clark, and P. Goodford, J. Med. Chem. 36, 140 (1993).

[5] E. Arunan, G. Desiraju, R. Klein, J. Sadlej, S. Scheiner, I. Alkorta, D. Clary, R. Crabtree, J. Dannenberg, P. Hobza, H. G. Kjaergaard, A. C. Legon, B. Mennucci, and D. J. Nesbitt, Pure Appl. Chem. 83, 1637 (2011).

[6] J. Teixeira and M. C. Bellissent-Funel, J. Phys. Condens. Mat. 2, SA105 (1990).

[7] A. Soper and M. Phillips, Chem. Phys. 107, 47 (1986).

[8] M. Matsumoto, J. Chem. Phys. 126, 054503 (2007).

[9] R. Kumar, J. R. Schmidt, and J. L. Skinner, J. Chem. Phys. 126, 204107 (2007).

[10] V. Buch, J. Chem. Phys. 96, 3814 (1992).

[11] A. Luzar and D. Chandler, Phys. Rev. Lett. 76, 928 (1996).

[12] A. D. Hammerich and V. Buch, J. Chem. Phys. 128, 111101 (2008).

[13] J. D. Smith, C. D. Cappa, K. R. Wilson, R. C. Cohen, P. L. Geissler, and R. J. Saykally, Proc. Natl. Acad. Sci. U.S.A. 102, 14171 (2005).

[14] R. H. Henchman and S. J. Irudayam, J. Phys. Chem. B 114, 16792 (2010).

[15] N. Agmon, Accounts Chem. Res. 45, 63 (2011).

[16] H. J. C. Berendsen, J. P. M. Postma, W. F. van Gunsteren, and J. Hermans, Intermolecular Forces 11, 331 (1981).

[17] H. J. C. Berendsen, J. R. Grigera, and T. P. Straatsma, J. Phys. Chem. 91, 6269 (1987).

[18] W. L. Jorgensen, J. Chandrasekhar, J. D. Madura, R. W. Impey, and M. L. Klein, J. Chem. Phys. 79, 926 (1983).

[19] W. L. Jorgensen and J. D. Madura, Mol. Phys. 56, 1381 (1985).

[20] H. W. Horn, W. C. Swope, J. W. Pitera, J. D. Madura, T. J. Dick, G. L. Hura, and T. Head-Gordon, J. Chem.
Phys. 120, 9665 (2004).

[21] J. L. F. Abascal and C. Vega, J. Chem. Phys. 123, 234505 (2005).

[22] W. Jorgensen, J. Chandrasekhar, J. Madura, R. Impey, and M. Klein, J. Chem Phys 79, 926 (1983).

[23] R. Shevchuk, D. Prada-Gracia, and F. Rao, J. Phys. Chem. B 116, 7538 (2012).

[24] D. Van Der Spoel, E. Lindahl, B. Hess, G. Groenhof, A. E. Mark, and H. J. C. Berendsen, J. Comput. Chem. 26, 1701 (2005)

[25] G. Bussi, D. Donadio, and M. Parrinello, J. Chem. Phys. 126, 014101 (2007).

[26] H. J. C. Berendsen, J. P. M. Postma, W. F. van Gunsteren, A. DiNola, and J. R. Haak, J. Chem. Phys. 81, 3684 (1984).

[27] T. Darden, D. York, and L. Pedersen, J. Chem. Phys. 98, 10089 (1993).

[28] F. W. Starr, J. K. Nielsen, and H. E. Stanley, Phys. Rev. Lett. 82, 2294 (1999).

[29] P. Geissler, C. Dellago, and D. Chandler, J. Phys. Chem. B 103, 3706 (1999).

[30] F. Rao and A. Caflisch, J. Mol. Biol. 342, 299 (2004).

[31] S. Krivov and M. Karplus, Proc. Natl. Acad. Sci. U.S.A. 101, 14766 (2004).

[32] F. Rao, S. Garrett-Roe, and P. Hamm, J. Phys. Chem. B 114, 15598 (2010).

[33] G. Berezovska, D. Prada-Gracia, S. Mostarda, and F. Rao, J. Chem. Phys 137, 194101 (2012).

[34] O. Markovitch and N. Agmon, J. Phys. Chem. A 111, 2253 (2007).

[35] V. Znamenskiy and M. Green, J. Chem. Theory Comput. 3, 103 (2007).

[36] W. C. Swope, J. W. Pitera, and F. Suits, J. Phys. Chem. B 108, 6571 (2004).

[37] J. Chodera, N. Singhal, V. Pande, K. Dill, and W. Swope, J. Chem. Phys. 126, 155101 (2007).

[38] D. Prada-Gracia, R. Shevchuk, P. Hamm, and F. Rao, J. Chem. Phys. 137, 144504 (2012). 